Check for updates

Cite this: RSC Adv., 2018, 8, 22777

Received 18th May 2018

Accepted 2nd June 2018

DOI: $10.1039 / \mathrm{c} 8 \mathrm{ra0} 4250 \mathrm{k}$

rsc.li/rsc-advances

\section{The assessment of the eco-toxicological effect of gabapentin on early development of zebrafish and its antioxidant system}

\author{
Xiuwen Li, ${ }^{a}$ Shuangxi Zhou, ${ }^{a}$ Yuting Qian, ${ }^{a}$ Zhuoran $X u,{ }^{a}$ Yang Yu, (D) ${ }^{a}$ Yanhua $\mathrm{Xu}{ }^{a}$ \\ Yide $\mathrm{He}^{\star a b}$ and Yongjun Zhang ${ }^{\star a}$
}

Gabapentin (GAB) is an emerging contaminant that is frequently detected in water bodies across the globe. The present study used zebrafish as a model organism to investigate the effects of GAB on the early development of zebrafish and on its antioxidant system. Acute toxicity tests indicated that the $96 \mathrm{~h} \mathrm{LC_{50 }}$ value of $\mathrm{GAB}$ for zebrafish embryos was $59.9 \mathrm{~g} \mathrm{~L}^{-1}$. Further, it was observed that GAB causes malformation of embryos such as hemagglutination and pericardial edema. Compared to the control group, a significant enhancement $(p<0.05)$ of heartbeat rates was found at GAB concentrations exceeding $50 \mathrm{mg} \mathrm{L}^{-1}$, while the swimming frequency was clearly increased upon exposure to GAB at a concentration of $100 \mathrm{mg} \mathrm{L}^{-1}(p<0.05)$. Additionally, the development of the zebrafish embryo was also negatively impacted after exposure to GAB as demonstrated by significantly decreased body lengths. Exposure to $\mathrm{GAB}$ at concentrations exceeding $50 \mathrm{mg} \mathrm{L}^{-1}$ significantly influenced the development of zebrafish, leading to malformation of organs and abnormal movements. Although no significant developmental effects of GAB were observed at environmentally relevant concentrations $(0.1$ and $10 \mu \mathrm{g} \mathrm{L}^{-1}$ ), further research about the antioxidant system confirmed that severe oxidant injury happened inside the organisms. catalase (CAT), lactate dehydrogenase (LDH), glutathione S-transferase (GST), glutathione (GSH) and the ability of inhibition of hydroxyl radicals (IHR) were used as biomarkers in the present study to illustrate GAB toxicity at environmentally relevant concentrations. The results showed that activities of CAT, LDH and GST as well as IHR were all elevated after GAB exposure, which proved that ROS were formed in the body as derived from GAB exposure. Among all of these biomarkers, CAT was the most sensitive one to evaluate the influence of $G A B$, and showed a significant increase even at a very low exposure concentration $\left(0.1 \mu \mathrm{g} \mathrm{L}^{-1}\right)$.

\section{Introduction}

The vast use of pharmaceuticals and personal care products (PPCPs) has caused their widespread distribution and frequent detection in the global aquatic environment. For example, researchers detected one or more of these chemicals in $80 \%$ of the American streams sampled during 1999-2000. Gabapentin (GAB) is one of the antiepileptic drugs, which are used for the treatment of partial seizures and neuropathic pain. ${ }^{1}$ In 2009, Germany consumed 58.9 t GAB, which increased to $73.3 \mathrm{t}$ in 2012. ${ }^{2,3} \mathrm{GAB}$ was also widely used in many Asian countries, like China, India, Singapore, and so on. ${ }^{4-6} \mathrm{GAB}$ is not easily metabolized in biota and the human excretion rate is therefore very high. ${ }^{7,8}$ When released to sewage treatment plants (STPs),

${ }^{a}$ School of Environmental Sciences and Engineering, Nanjing Tech University, Jiangsu, 211816, P. R. China. E-mail: heyd@njtech.edu.cn; y.zhang@njtech.edu.cn; Tel: +86-25-58139656

${ }^{b}$ Hunan Provincial Key Laboratory of Renewable Energy Electric-Technology, Changsha, Hunan, 410076, P. R. China a very low removal efficiency was often obtained. ${ }^{3}$ For instance, R. Gurke et al. detected 56 PPCPs in the influent and effluent of German STPs, and found that the removal efficiency of GAB was only 6.4\%. ${ }^{9}$ In Minnesota, researchers sampled 24 STP effluents, and many kinds of PPCPs were detected, among which the concentration of $\mathrm{GAB}$ varied in the ng $\mathrm{L}^{-1}$ range. ${ }^{\mathbf{1 0}}$ The low removal efficiency in STPs leads to its frequent detection in surface waters worldwide. In the UK and America, GAB has been detected at the concentration level of $n g \mathrm{~L}^{-1} \cdot .^{11,12}$ In Vidy Bay, Switzerland, GAB has been even detected at a concentration of $400 \mathrm{ng} \mathrm{L}^{-1}$ in raw drinking water. ${ }^{13}$ The frequent occurrence of $\mathrm{GAB}$ in aquatic systems raises concerns about its ecotoxicity, but to the best of our knowledge, hardly any studies on the ecotoxicity of GAB can be found in literature.

In the present study, zebrafish (Danio rerio) was used as a model animal. In comparison with traditional animal models, zebrafish offers many advantages, such as small size, short generation time, large numbers of eggs in one spawning, rapid development of embryos and so on. ${ }^{\mathbf{1 4 - 1 6}}$ In addition, the embryos and larvae of zebrafish are transparent, ${ }^{17,18}$ so it is 
relatively easy to observe adverse effects such as abnormalities induced by toxicants. In the recent 20 years, zebrafish has been widely used in toxicity tests. ${ }^{16-20}$ The fish embryo toxicity (FET), a standardized OECD test, is the most popular alternative means to live fish in acute test. ${ }^{21}$ In their whole life circle, embryo and larva stages are the most sensitive ones to chemicals. ${ }^{2-25}$ Before hatching, the embryos are surrounded by a chorion for about $72 \mathrm{~h}$, which may provide a protective barrier between the developing embryos and chemicals. ${ }^{2627}$ The present study extended the exposure period to $192 \mathrm{hpf}$ (hours post fertilization) covering more of the important developmental stages and thus providing more information about the ecotoxicity of GAB.

Fish exposed to the environmental contaminates exhibit sensitive antioxidant defences, which were often used as biological indicators of aquatic environmental health. ${ }^{28}$ The antioxidant enzymes, including catalase (CAT) and lactate dehydrogenase (LDH), represent a first line of defence against external oxidant stress, ${ }^{29}$ while the glutathione system, including glutathione S-transferase (GST) and glutathione (GSH) represent the second line. ${ }^{30}$ Besides, several nonenzymatic mechanisms can also indicate antioxidant defences, such as the ability of inhibition of hydroxyl radicals (IHR). ${ }^{31}$ The liver is where these antioxidant enzymes are produced in order to eliminate the reactive oxygen species (ROS) in organism. ${ }^{32}$ However, larvae at $96 \mathrm{hpf}$ as used in the present study were too tiny to isolated liver tissue, so whole larvae were used to prepare samples for the enzyme activity tests.

\section{Materials and methods}

\subsection{Chemicals}

GAB (CAS 60142-96-3, purity > 98\%) was purchased from Aladdin Industrial Corporation. NaCl (CAS 7647-14-5, purity > 99.5\%) was purchased from Xilong Scientific Co., Ltd. $\mathrm{CaCl}_{2}$ $\cdot 2 \mathrm{H}_{2} \mathrm{O}$ (CAS 10035-04-8, purity $>99.0 \%$ ) was purchased from Sinopharm Chemical Reagent Co., Ltd. $\mathrm{MgSO}_{4} \cdot 7 \mathrm{H}_{2} \mathrm{O}$ (CAS 10034-99-8, purity > 99.0\%), $\mathrm{NaHCO}_{3}$ (CAS 144-55-8, purity > 99.5\%) and $\mathrm{KCl}$ (CAS 7447-40-7, purity $>99.5 \%$ ) were purchased from Shanghai Lingfeng Chemical Reagent Co., Ltd. The CAT kit (Cat. no: KGT017), the GSH kit (Cat. no: KGT006), the GST kit (Cat. no: KGT005), the LDH kit (Cat. no: KGT02448), the $\cdot \mathrm{OH}$ kit (Cat. no: KGT010) and the Bradford protein quantitation assay kit (Cat. no: KGT801) were purchased from KeyGEN bio TECH Co., Ltd.

\subsection{Zebrafish husbandry and egg collection}

Adult wild-type zebrafish (Tübingen line) was purchased from Nanjing YSY Biotech Company, and acclimatized in our laboratory for at least one month before use in the experiments. The husbandry methods followed the standard guidance described in details elsewhere. ${ }^{33}$ Adult zebrafish were maintained in an incubator at $28 \pm 0.5^{\circ} \mathrm{C}$ with a $14 / 10 \mathrm{~h}$ light and dark cycle. They were fed three times daily with live baby brine shrimp for 5 minutes. On the day before spawning, one male and one female were transferred into the breeding box, and they were separated by means of a division plate. Next day, when the light period started, the division plate was removed, and after an hour, embryos were collected and washed several times with the embryonic culture medium water, containing $2 \mathrm{mM} \mathrm{CaCl}_{2}$ $\cdot 2 \mathrm{H}_{2} \mathrm{O}, 0.5 \mathrm{mM} \mathrm{MgSO} \cdot \cdot 7 \mathrm{H}_{2} \mathrm{O}, 0.75 \mathrm{mM} \mathrm{NaHCO}{ }_{3}$ and $0.08 \mathrm{mM}$ $\mathrm{KCl}$ (ISO 6341-1982). ${ }^{34}$

\subsection{Acute toxicity test}

It was reported that the NOEC value of GAB was $10 \mathrm{~g} \mathrm{~L}^{-1} .^{12}$ However, there was no acute toxicity testing to calculate the $\mathrm{LC}_{50}$ of GAB for zebrafish embryo. For general acute toxicity testing, embryos were exposed in test solutions, containing 50, $56,60,63,67,71 \mathrm{~g} \mathrm{~L}^{-1} \mathrm{GAB}$ for a 96 hours period starting from 12 hpf. This exposure concentrations were decided by a series pre-test. A negative control with water and a positive control with $4 \mathrm{mg} \mathrm{L}^{-1}$ 3,4-dichloroaniline were used in the present study. 12-well cell culture clusters (Corning Incorporated, made in USA) were chosen as the test chamber. 10 viable eggs were put in one well with $4 \mathrm{~mL}$ medium solution. Three duplicates were prepared for each test. The culture clusters were covered with lids to prevent evaporation during exposure, and kept in the incubator at a temperature of $28 \pm 0.5{ }^{\circ} \mathrm{C}, \mathrm{pH}$ at $7.11 \pm 0.05$, conductivity at $293.6 \pm 2.4 \mu \mathrm{s} \mathrm{cm}^{-1}$, and a light and dark cycle of $14 / 10$ hours. In this semi-static exposure test, $3 \mathrm{~mL}$ medium per well was renewed daily. Dead embryo were removed and recorded every 24 hours.

\subsection{Developmental toxicity test}

This experiment was designed and modified referring to the guideline of OECD TG 236, OECD TG 210 and OECD TG 212. ${ }^{35-37}$ The concentration gradient of GAB was set as $0.1 \mu \mathrm{g} \mathrm{L}^{-1}, 10 \mu \mathrm{g}$ $\mathrm{L}^{-1}, 0.1 \mathrm{mg} \mathrm{L} \mathrm{L}^{-1}, 1 \mathrm{mg} \mathrm{L} \mathrm{L}^{-1}, 10 \mathrm{mg} \mathrm{L}^{-1}, 50 \mathrm{mg} \mathrm{L}^{-1}$ and $100 \mathrm{mg} \mathrm{L}^{-1}$, plus the positive control and the negative control with no GAB. Three replicates were performed with every group. The cultivation condition were kept the same as in case of the acute toxicity test.

Embryos/larvae were observed at the following points in time: 6 hpf, 8 hpf, 12 hpf, 24 hpf, 48 hpf, 72 hpf, 96 hpf, 120 hpf, $144 \mathrm{hpf}, 168 \mathrm{hpf}, 192 \mathrm{hpf}$ with a biological inverted microscope (Nikon 120c, Japan) or a stereo microscope (Jiangnan JSZ6S, China) and photos were taken. Hatching rate was calculated daily. The apical performance of each tested embryos included: coagulation of embryos, no somite formation, non-detachment of the tail, and no heartbeat. The deformities included: pigmentations; deformity of yolk, pericardial edema; yolk sac edema; hemagglutination; tail deformation; swim bladder defects. ${ }^{38,39}$

In order to observe the effects of GAB on the development of zebrafish, several endpoints were used to identify the variation between test groups and control group, as shown in Table $1 .^{\mathbf{4 0 , 4 1}}$

\subsection{Biomarkers in antioxidant system test}

From $12 \mathrm{hpf}$ to $96 \mathrm{hpf}$, embryos in the tested groups were exposed to $0.1 \mu \mathrm{g} \mathrm{L}^{-1}, 10 \mu \mathrm{g} \mathrm{L}^{-1}$ and $1 \mathrm{mg} \mathrm{L}^{-1} \mathrm{GAB}$, representing a realistic environmental concentration, the worst case environmental level and a pharmaceutically relevant concentration 
Table 1 Endpoints used in the present study

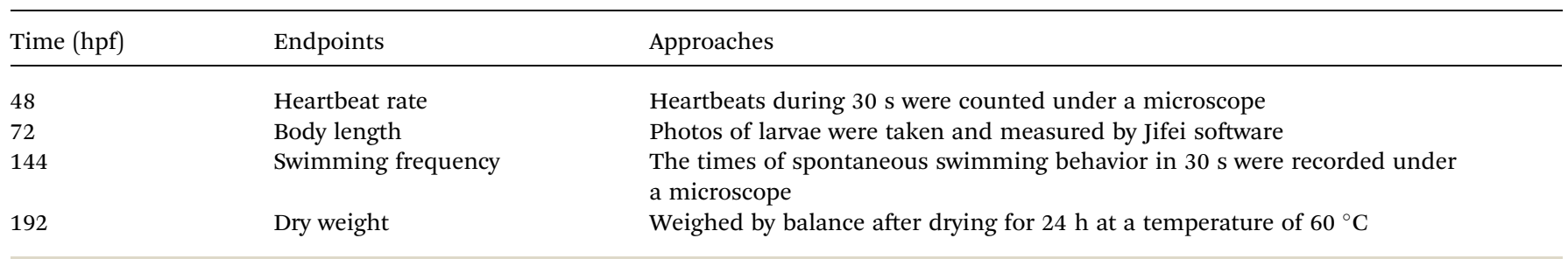

respectively. Three replicates were used in every group. The cultivation conditions were kept the same as those for the acute toxicity tests described above. At the end of exposure, $96 \mathrm{hpf}$ larvae and embryos were killed on ice, homogenized in $0.6 \%$ $\mathrm{NaCl}$ using a Hand-held cell crusher (MP FastPrep-1, America), and centrifuged at $6000 \mathrm{rpm}$ for $10 \mathrm{~min}$ at $4{ }^{\circ} \mathrm{C}$. The supernatants were collected for enzyme testing following the instructions of those kits.

\subsection{Statistical analyses}

All data were analyzed with PASW Statistics 18, including the focus on one-way ANOVA of $48 \mathrm{hpf}$ heart beats; $72 \mathrm{hpf}$ body length; $144 \mathrm{hpf}$ swimming frequency; $192 \mathrm{hpf}$ dry weight as well as all kinds of antioxidant biomarkers. The significance level was set at $p<0.05$.

\subsection{Live subject statement}

This study was performed in strict accordance with the Laboratory Animal-Guideline for ethical review of animal welfare (GB/T 35 892-2018), and was approved by Animal Care and Use Committee in School of Environmental Science and Technology of Nanjing Tech University (Nanjing, China).

\section{Results and discussion}

\subsection{Mortality and $\mathrm{LC}_{50}$}

The mortality of zebrafish was assessed once every $24 \mathrm{~h}$. The dead embryos mostly showed coagulation, which was followed by a lack of somite formation or heartbeat while no embryo showed non-detachment of the tail (Fig. 1).

In the acute toxicity test, all of embryos exposed to $67 \mathrm{~g} \mathrm{~L}^{-1}$ GAB died during $96 \mathrm{~h}$ exposure, while the negative control and the positive control own the death rate of 0 and $100 \%$. This result validated the quality of embryos. After $96 \mathrm{~h}$ exposure, $\mathrm{LC}_{50}$ of $59.9 \mathrm{~g} \mathrm{~L}^{-1}$ was calculated (Fig. 2).
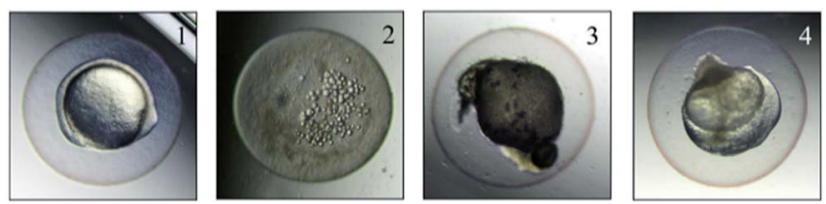

Fig. 1 Appearance of dead embryos: (1) normal, (2) coagulation, (3) lack of somite formation, (4) lack of heartbeat.

\subsection{Heartbeat rates and movements}

The effects of GAB on the heartbeat rate and the swimming frequency of zebrafish embryos and larvae are illustrated in Fig. 3. A clear increment of heartbeat rate can be found along the increasing concentrations of GAB (Fig. 3(a)). A significant ( $p$ $<0.05$ ) increment appeared at the exposure level of $50 \mathrm{mg} \mathrm{L}^{-1}$ $\mathrm{GAB}$, resulting in $5.1 \%$ increase as compared to the control group. The increment of heartbeat rates became more severe at the highest concentration of $100 \mathrm{mg} \mathrm{L}^{-1}$.

In the developmental process of zebrafish, heart is the first organ to form, which was visible because of the transparent development of zebrafish. ${ }^{42}$ The heartbeat of zebrafish develops to be regular at around $36 \mathrm{hpf}^{43}$ and this parameter is directly linked to the temperature. ${ }^{44}$ The values measured in this study were determined at $30{ }^{\circ} \mathrm{C}$ and at $48 \mathrm{hpf}$. The heartbeat rate in the control group is about $72 \pm 1.6$ beats per $30 \mathrm{~s}$, and showed a distinguishable tendency of increasing with the exposure concentration, which indicated that heartbeat rate can be used as a sensitive indicator in this test.

Within ecotoxicity assessment of drugs the heartbeat rate at $48 \mathrm{hpf}$ is often used as an indicator to assess adverse effects of pollutants, and various publications showed significant changes in tested groups. ${ }^{\mathbf{4 3 4}}$ Interestingly, another two psychotropic drugs, carbamazepine and valproic acid commonly used for the treatment of epilepsy, induced a significant and concentration-dependent decrease of the heartbeat rate in a laboratory test, ${ }^{\mathbf{4 6}}$ which was exactly opposite to the result of the present study. It was reported that when rats were exposed to gabapentin, the heartbeat rate was elevated too. ${ }^{47}$ This finding showed that GAB not only increased the heartbeat rate of zebrafish, but also of mammals.

The swimming frequency was slightly increased from $10 \mathrm{mg} \mathrm{L}^{-1}$ to $100 \mathrm{mg} \mathrm{L}^{-1}$, and showed a significant elevation at

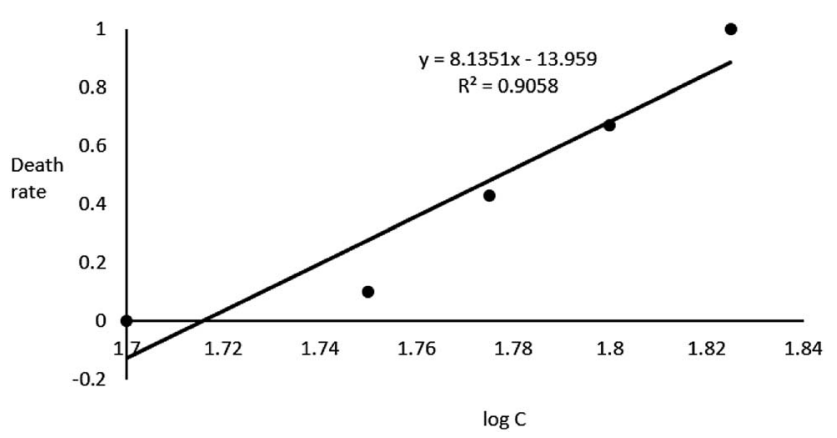

Fig. 2 Correlation between zebrafish embryo death rate and $\log C$. 

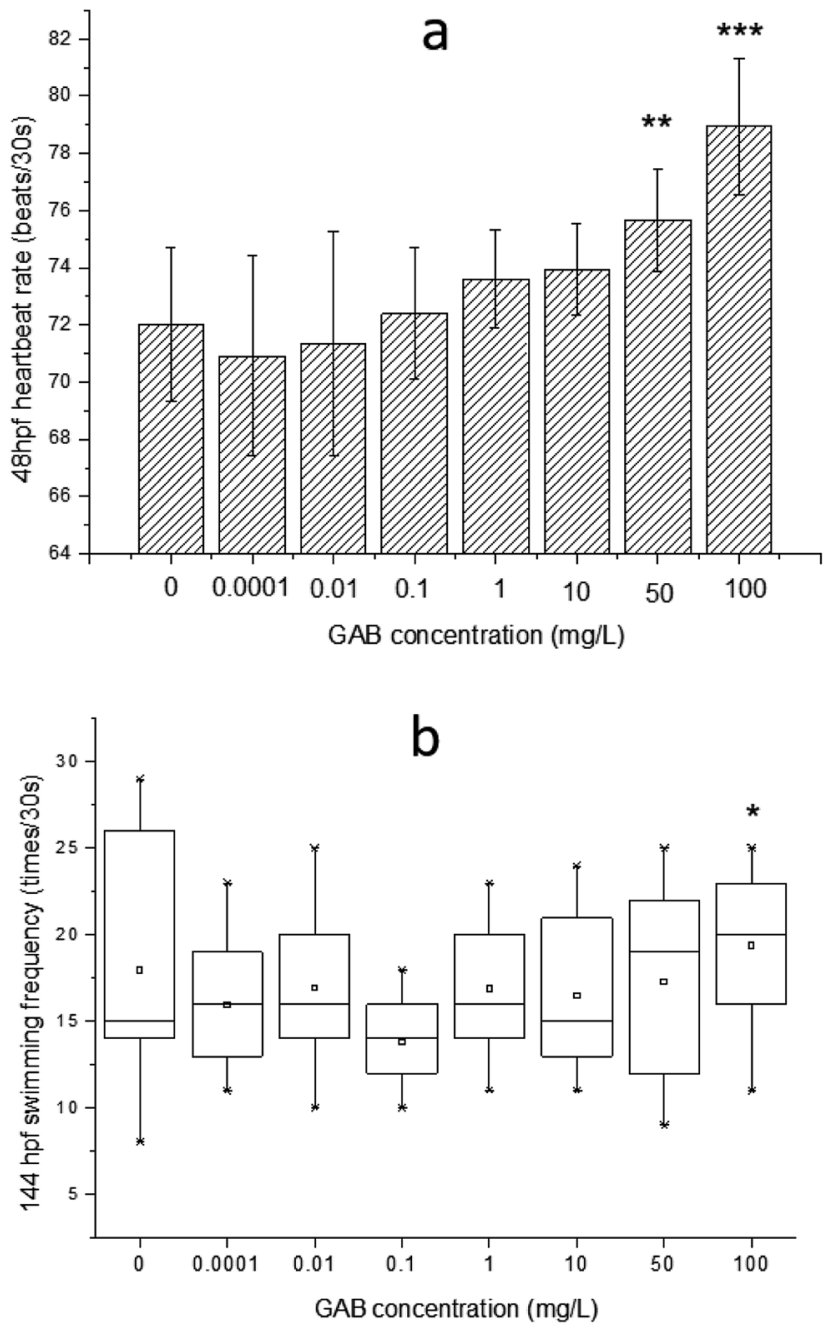

Fig. 3 (a) The effect of GAB on the heartbeat rate at $48 \mathrm{hpf}$ of zebrafish embryos, (b) swimming frequency at 144 hpf of zebrafish larvae exposed to GAB of different concentrations. The result of one-way ANOVA is $* p<0.05, * * p<0.01$ and $* * * p<0.001$.

$100 \mathrm{mg} \mathrm{L}^{-1}(p<0.05)$. Testing of fish behavior is a great tool for ecotoxicological studies, because it interprets the exerted complex physiological effects of pollutants at the individual level. ${ }^{48}$ Among different kinds of behavior of zebrafish, swimming plays an important role including swimming speed, acceleration, routine turns, swimming bouts, the shape of body wave and swimming frequency. ${ }^{49-51}$ In the present study, the swimming frequency was measured at $144 \mathrm{hpf}$. At this time point, larvae became active and the swimming behavior were easy to be observed. The result of the test showed a whole tendency upon increasing exposure concentration, which is consistent with the result of the heartbeat rate test. It seems that GAB activated zebrafish and this might be caused by the mechanism action of GAB to the nervous system of zebrafish. ${ }^{52}$ In the drug toxicity testing of gabapentin, Wolf et $a .^{53}$ found that the children became hyperactive and explosive outbursts consisting of aggressive and oppositional behavior.

\subsection{Body length and dry weight}

The effect of GAB on the body length of zebrafish is illustrated in Fig. 4. There was no significant decent in body length, but a reducing tendency was observable. The body length was reduced by $2.7 \%$ at $10 \mathrm{mg} \mathrm{L}^{-1}, 2.0 \%$ at $50 \mathrm{mg} \mathrm{L}^{-1}$, and $2.8 \%$ at $100 \mathrm{mg} \mathrm{L}^{-1}$, as compared with the control group. Nevertheless, the dry weight of larvae of all test groups showed no significant changes: $100 \pm 6.7 \mu \mathrm{g}$ per larva.

Body length is associated with risk-related behavior in zebrafish, ${ }^{54}$ and it is a useful and intuitional parameter in the experiment. Carbamazepine, an antiepileptic drug, which has a longer history in human use than GAB, induced an increase of body length. ${ }^{55}$ However, in the present study, exposure to GAB caused a concentration-dependent decrease of body length of zebrafish at $72 \mathrm{hpf}$. This strongly indicates that GAB has adverse effects on the development of zebrafish. Besides body length, Fraysse et al. used a similar parameter, tail length, to assess toxicity of chemicals. The result of their study illustrated tail length is shorten following exposure to pollutants. ${ }^{56}$ Dry weight as another important physiological index was measured at 192 hpf, the end of the exposure period. The dry weight showed no obvious difference among the control group and all test groups. This might partly be due to the fact that the animals were not fed during the whole experiment.

\subsection{Morphology of the embryos}

The morphology of zebrafish from the embryos to the juveniles' development stage was observed at $6 \mathrm{hpf}, 8 \mathrm{hpf}, 12 \mathrm{hpf}, 24 \mathrm{hpf}$, $48 \mathrm{hpf}$ and $192 \mathrm{hpf}$. Only up till $48 \mathrm{hpf}$, morphological malformations were observed in the tested groups. The physical appearance before $24 \mathrm{hpf}$ is shown in Fig. 5 .

At $48 \mathrm{hpf}$, malformations, including pericardial edema and hemagglutination were visible, whereas pericardial edema was the common malformation in the present study, that was observed in every test group (Fig. 6). Pericardial edema is the most popular indicator in zebrafish toxicity test, which is for

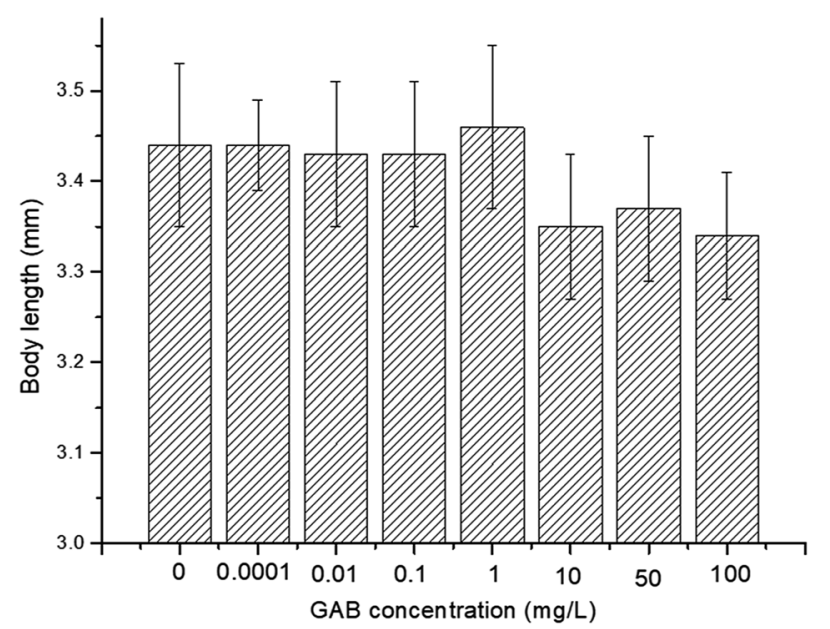

Fig. 4 Body length of 72 hpf zebrafish exposed to different concentrations of GAB. 

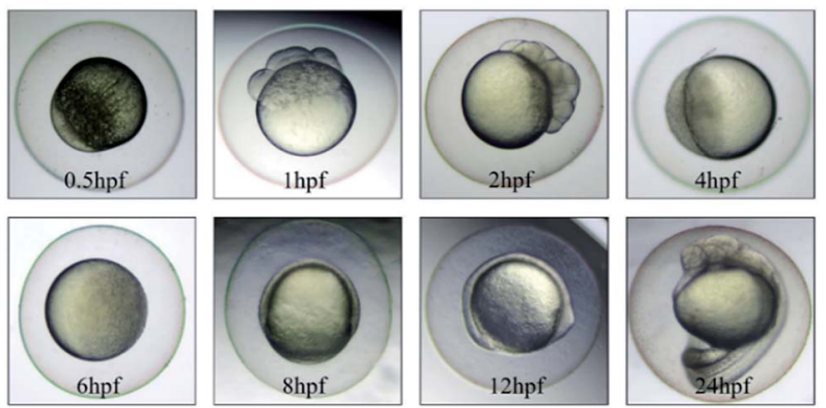

Fig. 5 The physical appearance of zebrafish embryos from $0.5 \mathrm{hpf}$ to $24 \mathrm{hpf}$.
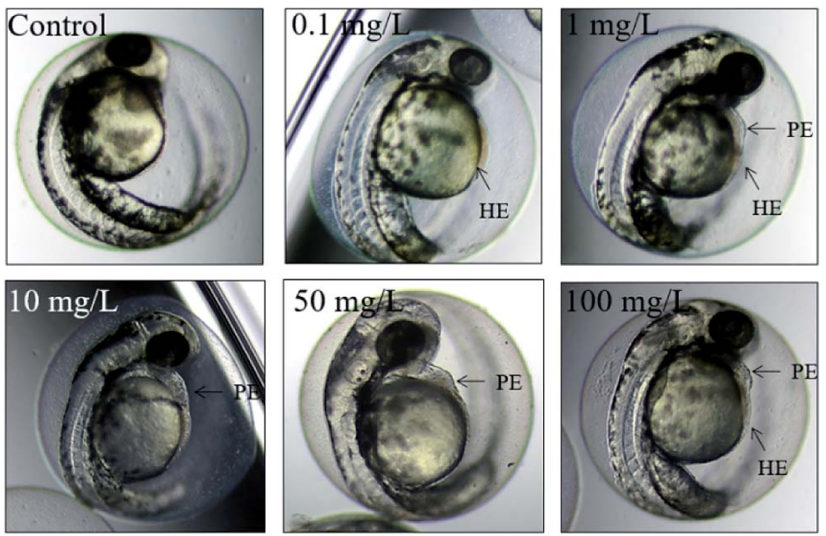

Fig. 6 The malformations of zebrafish at $48 \mathrm{hpf}$. HE: hemagglutination, PE: pericardial edema.

instance also deemed as the most sensitive indicator for ethanol exposure in zebrafish. ${ }^{57}$

\subsection{Antioxidant biomarkers}

ROS was produced during the use of oxygen in the metabolism process, among which hydroxyl radicals $(\cdot \mathrm{OH})$ were deemed as being the most damaging ROS. ${ }^{29}$ The activity of IHR in the body can indirectly reflect the content of $\cdot \mathrm{OH}$ and explain the oxidative stress derived from environmental pollutants. In the present study, after exposure, the IHR in zebrafish was significantly elevated at $10 \mu \mathrm{g} \mathrm{L}^{-1}(p<0.01)$ and $1 \mathrm{mg} \mathrm{L}^{-1}(p<0.001)$, as shown in Fig. 7. This results indicated that when the zebrafish exposed to oxidative stress, they tried to protect themselves from tissue injury by increasing the activity of antioxidant enzymes as a compensatory mechanism.

CAT can convert $\mathrm{H}_{2} \mathrm{O}_{2}$ to $\mathrm{H}_{2} \mathrm{O}$ and $\mathrm{O}_{2}$ very efficiently, so accumulation of superoxide chemicals will enhance CAT activity.$^{58}$ Compared to the control groups, the activities of CAT in all test groups were elevated, and the higher the exposure concentration, the higher CAT activity. Even at the lowest concentration $\left(0.1 \mu \mathrm{g} \mathrm{L}^{-1}\right)$ the change was highly significant $(p<$ 0.001), as shown in Fig. 8(a).

$\mathrm{LDH}$ is an essential enzyme in the anaerobic pathway of energy production, and can indicate oxidative stress, so that

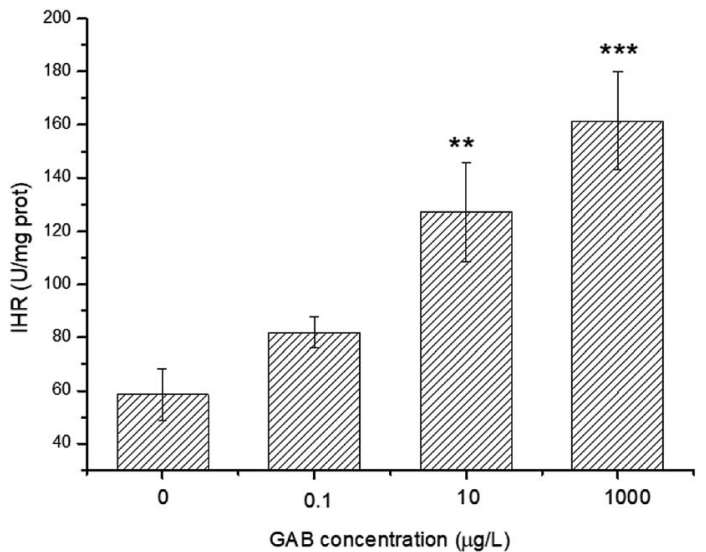

Fig. 7 IHR in zebrafish at $96 \mathrm{hpf}$. The results of one-way ANOVA are indicated at the significance levels $* * p<0.01$ and $* * * p<0.001$.

often be used as a biomarker for evaluate toxicity of chemicals. ${ }^{59}$ The activities of $\mathrm{LDH}$ in zebrafish exposed to environmentally relevant concentrations GAB were mainly at the same level as in the control groups. However, when the concentration was increased up to $1 \mathrm{mg} \mathrm{L^{-1 }}$, activity of $\mathrm{LDH}$ was significant increased ( $p<0.001)$, as shown in Fig. 8(b).

GSH is an essential reducing chemical, whose concentration is always regulated at a stable level when the antioxidant system
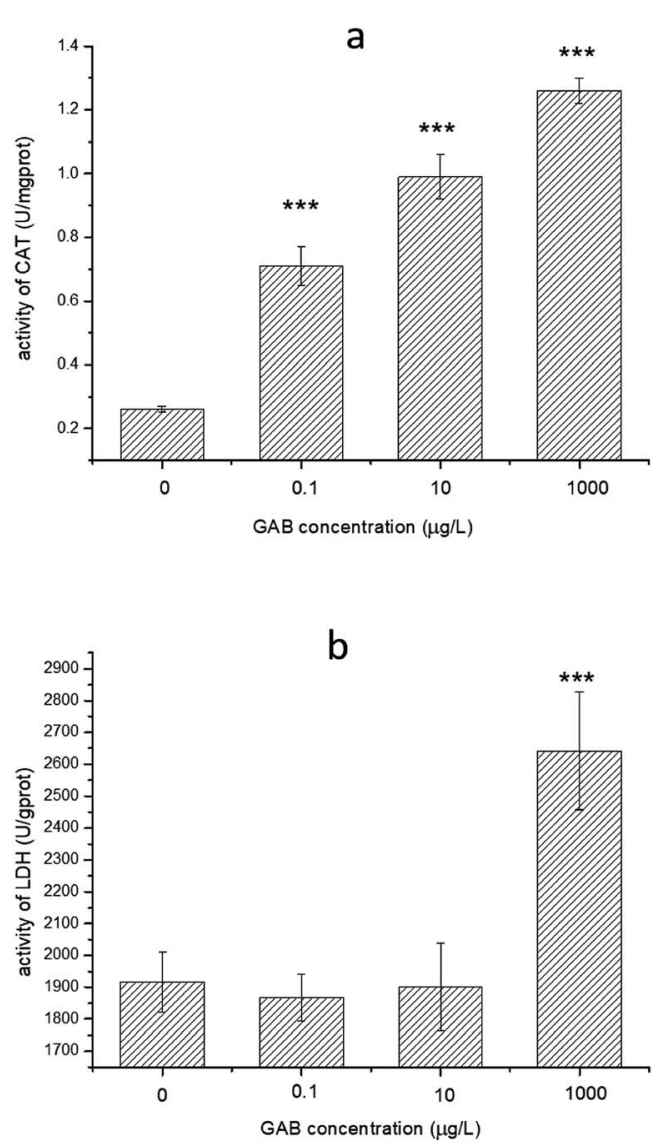

Fig. 8 Activity of CAT and LDH in zebrafish at $96 \mathrm{hpf}$. The result of one-way ANOVA is in all cases: ***p $<0.001$. 
is normal. ${ }^{60}$ When organisms face oxidant pressure, overwhelming amount of oxidizing substances will be formed. GST can convert these harmful substances with GSH to fulfil detoxification. Besides, GST can also reduce organic peroxides. ${ }^{61}$ As shown in Fig. 9, the activity of GST was significantly increased after GAB exposure, especially at high exposure concentrations. At the lowest concentration, such elevation was not obvious, when the concentration was however increased to the worst case environmental level of $10 \mu \mathrm{g} \mathrm{L}^{-1}$, this change became remarkable and kept increasing.

Environmental pollution may induce the production of ROS, which will further cause the elevation of antioxidant enzymes as a defense mechanism. The present study demonstrated that the ability of IHR, and the activity of CAT, LDH and GST were enhanced by the treatment of GAB. The ability of IHR demonstrated that mass $\cdot \mathrm{OH}$ formed in the body. To avoid oxidant injury, the anti-oxidant system took measures to scavenge so many $\cdot \mathrm{OH}$. CAT was the most sensitive indicator in the present study, and showed a significant increase even at a very low concentration $\left(0.1 \mu \mathrm{g} \mathrm{L}^{-1}\right)$. The enhanced CAT enzymatic activity reflects the oxidative stress that occurred in fish. To withstand such stress, the anti-oxidative capacity of zebrafish
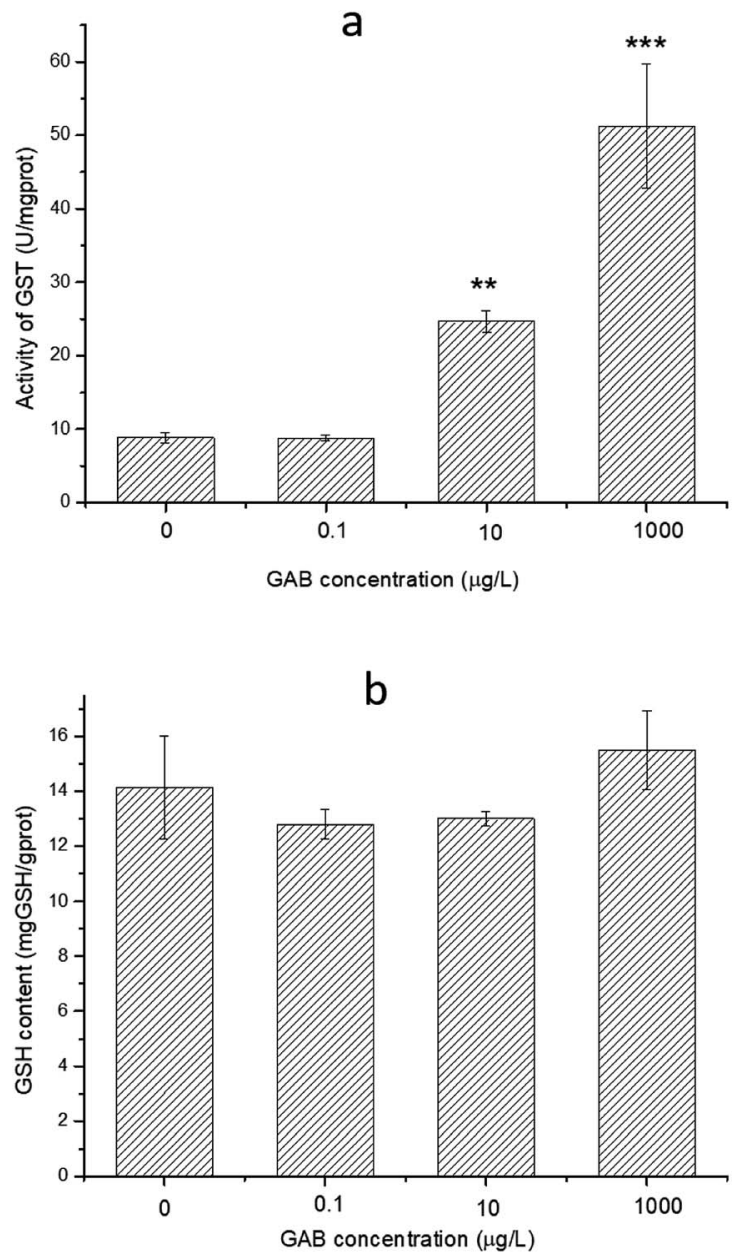

Fig. 9 Activity of GST and content of GSH in zebrafish at $96 \mathrm{hpf}$. The results of one-way ANOVA are ${ }^{* *} p<0.01$ and $* * * p<0.001$. was activated. Carbamazepine caused the elevation of CAT activity in rainbow trout after long term exposure. ${ }^{62}$ Valproic acid is commonly used for the treatment of epilepsy, and was found to inhibit the CAT activity in rat, which contradicts the results of the present study. ${ }^{63}$ Opposed to CAT, the change of $\mathrm{LDH}$ activity was not statistically significant in environmental concentration level used in this study. LDH activity is however still a key indicator of the oxidative stress as for instance shown in a study with the fresh water fish, Cyprinus carpio L. the toxicity of carbamazepine $\left(5.97 \mathrm{mg} \mathrm{L}^{-1}\right)$ was tested and in which it was found that the LDH activity was significantly increased. ${ }^{64}$ Elevated LDH activity suggests that the aerobic catabolism of glycogen and glucose has shifted towards the formation of lactate, which may induced toxicity to fish. ${ }^{65}$ Increased LDH activity also influences the muscles, which may indicate damage with regard to heartbeat rate. ${ }^{66}$ The heartbeat rate and the swimming frequency recoded at $48 \mathrm{hpf}$ and $144 \mathrm{hpf}$ were all elevated, which may have an underlying connection with LDH. To protect the body from the damage induced by pollutants, the activity of GST is usually increased, ${ }^{67}$ and the present study confirmed that GST activity is closely related to the exposure concentration. Elevated GST is able to eliminate harmful substances in the body with GSH.

\section{Conclusions}

The present study found that the $96 \mathrm{~h} \mathrm{LC}_{50}$ value of $\mathrm{GAB}$ was $59.9 \mathrm{~g} \mathrm{~L}^{-1}$, so $\mathrm{GAB}$ does not induce acute toxic effects at low exposure concentrations as neither mortality nor malformation of zebrafish embryos. Parameters like heartbeat rate and body length illustrated however that $\mathrm{GAB}$ is capable of inducing developmental toxicity to zebrafish embryos. GAB can affect the cardiac function, and the body length of zebrafish. Further research about the antioxidant system of zebrafish found that even at a very low concentration of GAB leads to oxidant injury in zebrafish. CAT, GST and IHR were found to be sensitive biomarkers in such toxicity tests. LDH not only indicated the effect of GAB on the antioxidant system, but is also able to clarify the mechanism of enhancement of heartbeat rate and swimming frequency. These results draw our attention to possible adverse effects of GAB in the environment and help scientists as well as regulators to prepare measures to reduce the adverse environmental effects of GAB.

\section{Conflicts of interest}

There are no conflicts to declare.

\section{Acknowledgements}

This work was financially supported by Natural Science Foundation of Jiangsu Province (No. BK20160989), Hunan Provincial Key Laboratory of Renewable Energy Electric-Technology (No. 2016ZNDL003), Major Research Program of Natural Science of University in Jiangsu Province (No. 16KJA610002), and Cultivation Project for International Cooperation from Nanjing Tech 
University. Thanks Prof. Dr Ir. Willie Peijnenburg from Leiden University for reviewing this manuscript.

\section{References}

1 M. C. Salinsky, D. Storzbach, D. C. Spencer, B. S. Oken, T. Landry and C. B. Dodrill, Neurology, 2005, 64, 792-798.

2 F. Y. Lai, C. Ort, C. Gartner, S. Carter, J. Prichard, P. Kirkbride, R. Bruno, W. Hall, G. Eaglesham and J. F. Mueller, Water Research, 2011, 45, 4437-4448.

3 M. Herrmann, J. Menz, O. Olsson and K. K Mmerer, Water Research, 2015, 85, 11-21.

4 A. C. F. Hui and P. Kwan, Seizure, 2004, 13, 244-246.

5 S. V. Thomas, P. S. Sarma, M. Alexander, L. Pandit, L. Shekhar, C. Trivedi and B. Vengamma, Epilepsia, 2001, 42, 1052-1060.

6 T. L. Mac, D. Tran, F. Quet, P. Odermatt, P. Preux and C. T. Tan, Lancet Neurol., 2007, 6, 533-543.

7 B. Kasprzyk-Hordern, R. M. Dinsdale and A. J. Guwy, Water Research, 2008, 42, 3498-3518.

8 J. T. Yu, E. J. Bouwer and M. Coelhan, Agric Water Manag., 2006, 86, 72-80.

9 R. Gurke, M. R Ler, C. Marx, S. Diamond, S. Schubert, R. Oertel and J. Fauler, Sci. Total Environ., 2015, 532, 762770.

10 J. H. Writer, I. Ferrer, L. B. Barber and E. M. Thurman, Sci. Total Environ., 2013, 461-462, 519-527.

11 B. Petrie, R. Barden and B. Kasprzyk-Hordern, Water Research, 2015, 72, 3-27.

12 R. P. Deo, Curr. Environ. Health Rep., 2014, 1, 113-122.

13 B. Morasch, F. Bonvin, H. Reiser, D. Grandjean, L. F. De Alencastro, C. Perazzolo, N. Chèvre and T. Kohn, Environ. Toxicol. Chem., 2010, 29, 1658-1668.

14 D. Li, C. Lu, J. Wang, W. Hu, Z. Cao, D. Sun, H. Xia and X. Ma, Aquat. Toxicol., 2009, 91, 229-237.

15 Z. Xu, F. E. Williams and M. Liu, J. Appl. Toxicol., 2011, 31, 157-163.

16 X. Gao, K. Li, L. Jiang, M. He, C. Pu, D. Kang and J. Xie, J. Appl. Toxicol., 2017, 37, 602-610.

17 J. Wixon, Yeast, 2000, 17, 225-231.

18 V. Tropepe and H. L. Sive, Genes, Brain Behav., 2003, 268281.

19 S. Scholz, S. Fischer, U. G Ndel, E. K Ster, T. Luckenbach and D. Voelker, Environ. Sci. Pollut. Res., 2008, 15, 394-404.

20 W. Zhang, K. Lin, Y. Miao, Q. Dong, C. Huang, H. Wang, M. Guo and X. Cui, J. Hazard. Mater., 2012, 213-214, 413420.

21 E. Lammer, G. J. Carr, K. Wendler, J. M. Rawlings, S. E. Belanger and T. Braunbeck, Comp. Biochem. Physiol., Part C: Toxicol. Pharmacol., 2009, 149, 196-209.

22 H. W. Laale and W. Lerner, Am. Zool., 1981, 21, 517-533.

23 Z. Lele and P. H. Krone, Biotechnol. Adv., 1996, 14, 57-72.

24 X. Shi, Y. Du, P. K. S. Lam, R. S. S. Wu and B. Zhou, Toxicol. Appl. Pharmacol., 2008, 230, 23-32.

25 K. Van den Bulck, A. Hill, N. Mesens, H. Diekman, L. De Schaepdrijver and L. Lammens, Reprod. Toxicol., 2011, 32, 213-219.
26 J. Chen, H. Gao, Y. Zhang, Y. Zhang, X. Zhou, C. Li and H. Gao, Sci. Rep., 2015, 4(4841), 1-7.

27 K. E. Pelka, K. Henn, A. Keck, B. Sapel and T. Braunbeck, Aquat. Toxicol., 2017, 185, 1-10.

28 J. Sturve, B. C. Almroth and L. Förlin, Ecotoxicol. Environ. Saf., 2008, 70, 446-452.

29 C. Rodriguez, J. C. Mayo, R. M. Sainz, I. Antolin, F. Herrera, V. Martin and R. J. Reiter, J. Pineal Res., 2004, 36, 1-9.

$30 \mathrm{M}$. Wu, H. Xu, Y. Shen, W. Qiu and M. Yang, Environ. Toxicol. Chem., 2011, 30, 2335-2341.

31 A. Prieto, A. Jos, S. Pichardo, I. Moreno and A. Cameam, Aquat. Toxicol., 2006, 77, 314-321.

32 I. Moreno, S. Pichardo, A. Jos, L. Gómez-Amores, A. Mate, C. M. Vazquez and A. M. Cameán, Toxicon, 2005, 45, 395402.

33 M. Westerfield, The Zebrafish Book, Institute of Neuroscience, University of Oregon, 1995.

34 L. Qiang, J. Cheng, J. Yi, J. M. Rotchell, X. Zhu and J. Zhou, Ecotoxicology, 2016, 25, 1426-1437.

35 OECD, Fish Embryo Acute Toxicity (FET) Test, OECD Guidelines for the Testing of Chemicals, 2013, vol. 236.

36 OECD, Fish Early-life Stage Toxicity Test, OECD Guidelines for the Testing of Chemicals, 2013, vol. 210.

37 OECD, Fish Short-term Toxicity Test on Embryo and Sac-fry Stages, OECD Guidelines for the Testing of Chemicals, 1998, vol. 212.

38 E. van den Brandhof and M. Montforts, Ecotoxicol. Environ. Saf., 2010, 73, 1862-1866.

39 T. Lin, Y. Chen and W. Chen, Environ. Toxicol. Pharmacol., 2013, 36, 1068-1076.

40 M. Strmac and T. Braunbeck, Ecotoxicol. Environ. Saf., 1999, 44, 25-39.

41 C. B. Kimmel, W. W. Balllard, S. R. Kimmel, B. Ullmann and T. F. Schilling, Dev. Dyn., 1995, 230, 255-310.

42 E. De Luca, G. M. Zaccaria, M. Hadhoud, G. Rizzo, R. Ponzini, U. Morbiducci and M. M. Santoro, Sci. Rep., 2015, 4(4849), 1-13.

43 B. Fraysse, R. Mons and J. Garric, Ecotoxicol. Environ. Saf., 2006, 63, 253-267.

44 W. R. Barrionuevo and W. W. Burggren, Am. J. Physiol., 1999, 276, R505-R513.

45 R. Nagel, ALTEX, 2002, 19, 38-48.

46 B. Pruvot, Y. Quiroz, A. Voncken, N. Jeanray, A. Piot, J. A. Martial and M. Muller, Reprod. Toxicol., 2012, 34, 568583.

47 M. H. Yoon and J. I. Choi, J. Korean Med. Sci., 2003, 18, 478482.

48 G. Ašmonaitè, S. Boyer, K. B. D. Souza, B. Wassmur and J. Sturve, Aquat. Toxicol., 2016, 173, 143-153.

49 S. A. Budick and D. M. O. Malley, J. Exp. Biol., 2000, 203, 2565-2579.

50 U. K. Muller, J. Exp. Biol., 2004, 207, 853-868.

51 D. A. Ritter, D. H. Bhatt and J. R. Fetcho, J. Neurosci., 2001, 21, 8956-8965.

52 A. Kukkar, A. Bali, N. Singh and A. S. Jaggi, Arch. Pharmacal Res., 2013, 36, 237-251. 
53 S. M. Wolf, S. Shinnar, H. Kang, K. B. Gil and S. L. Moshe, Epilepsia, 1996, 36, 1203-1205.

54 G. Polverino, D. Bierbach, S. S. Killen, S. Uusi-Heikkilä and R. Arlinghaus, J. Fish Biol., 2016, 1-17.

55 T. V. Madureira, M. J. Rocha, C. Cruzeiro, I. Rodrigues, R. A. F. Monteiro and E. Rocha, Environ. Toxicol. Pharmacol., 2012, 34, 34-45.

56 B. Fraysse, R. Mons and J. Garric, Ecotoxicol. Environ. Saf., 2006, 63, 253-267.

57 M. J. Reimers, A. R. Flockton and R. L. Tanguay, Neurotoxicol. Teratol., 2004, 26, 769-781.

58 J. M. Mates, C. P. Gomez and I. N. D. Castro, Clin. Biochem., 1999, 32, 595-603.

59 Z. Li, V. Zlabek, J. Velisek, R. Grabic, J. Machova, J. Kolarova, P. Li and T. Randak, Ecotoxicol. Environ. Saf., 2011, 74, 319327.
60 E. I. Stephensen, J. Sturve and L. F. Rlin, Comp. Biochem. Physiol., Part C: Toxicol. Pharmacol., 2002, 133, 435-442.

61 W. B. Jakobyz and D. M. Ziegler, J. Biol. Chem., 1990, 265, 20715-20718.

62 Z. Li, J. Velisek, V. Zlabek, R. Grabic, J. Machova, J. Kolarova and T. Randak, Chem.-Biol. Interact., 2010, 183, 98-104.

63 A. J. Akindele, E. Otuguor, D. Singh, D. Ota and A. S. Benebo, Eur. J. Pharmacol., 2015, 762, 174-183.

64 A. Malarvizhi, C. Kavitha, M. Saravanan and M. Ramesh, J. King Saud Univ., Sci., 2012, 24, 179-186.

65 T. Szegleyes, C. Polyhos, T. Balint, A. A. Rady, G. Lang, O. Kufcsak and J. Nemcsok, Environ. Monit. Assess., 1994, 35, 97-111.

66 J. Nemcsók, Á. Németh, Z. Buzás and L. Boross, Aquat. Toxicol., 1984, 5, 23-31.

67 S. Yan, J. Wang, L. Zhu, A. Chen and J. Wang, Ecotoxicol. Environ. Saf., 2015, 122, 54-60. 